\title{
Exploring the Societal Burden of Endometriosis on Women: a Meta-Ethnography
}

Olivia C. Nwankudu1; Israel Oluwaseyidayo Idris ${ }^{2,3}$; Francis Ifeanyi Ayomoh ${ }^{4}$; Uju Onyes ${ }^{5}$

${ }^{1}$ Department of Research and Advocacy, EndoSurvivors International Foundation

${ }^{2}$ Department of Social and Preventive Medicine, V.N Karazin Kharkiv National University, Kharkiv, Ukraine.

${ }^{3}$ Health Policy and Governance Unit, Department of State Management and Public Administration, Kharkiv National University of Economics, Ukraine.

${ }^{4}$ Nuffield Department of Primary Care Health Sciences, University of Oxford, United Kingdom.

${ }^{5}$ Department of Health Policy, London School of Economics and Political Science (LSE) London, United Kingdom.

Corresponding author: Olivia C. Nwankudu, olivia@endosurvivors.org 


\section{KEY POINTS}

Question: This study investigates the illness experience of endometriosis patients across the globe, in a bid to understand the societal impact of the disease on the lives and overall well-being of patients.

Finding: Illness experience was captured in nine categories through a conceptual model: disease symptoms; health services experience; isolation and limited social participation; limited physical functioning; a coterie of emotions; sex and intimate relationships; infertility; work life and education; coping strategies and support.

Meaning: Women with endometriosis appear to have similar experiences regardless of their nationalities and countries of residence. Endometriosis has a negative impact across all aspects of life, as reflected among nine areas of illness experience uncovered in this review. More research is needed to estimate the societal cost of the disease to capacitate the health system governance in providing better care and support for patients. 


\section{ABSTRACT}

Background: Endometriosis affects between $1 \%$ to $10 \%$ of women worldwide; it is associated with a significant burden on the woman, her relationships, productivity, mental health, family and society.

Aim: To contribute to the current understanding of the impact of endometriosis on women's lives by integrating qualitative research findings to explore the illness experiences of women living with endometriosis.

Study design: Synthesis of qualitative data using Britten's meta-ethnography.

Methods: Six bibliographic databases (Medline, Embase, CINAHL, Web of Science, Scopus and PsycINFO) and Google Scholar were searched for peer-reviewed papers published in English language from inception of the respective databases until August 6th 2021.

Results: Fifteen studies were included in the data synthesis, which comprised the only published study from the African continent which met the inclusion criteria. The review included a total of 354 women from fourteen countries (including South Africa, Iran, England, Australia, United States of America, Sweden, Brazil, China, India, New Zealand), of age range 16-78 years. Subjects represented diverse ethnicities, cultures and native languages distributed across socioeconomic classes. Representation of all stages of endometriosis was included.

Findings incorporate the following nine categories into a conceptual model: disease symptoms; health services experience; isolation and limited social participation; limited physical functioning; a coterie of emotions; sex and intimate relationships; infertility; work life and education; coping strategies and support. Diagnostic delays, persistent symptoms, inadequate education to patients about the disease, unsatisfactory services, healthcare costs and the absence of support, all intersect into a web of struggles and incoherence for patients.

Conclusion: Women with endometriosis living in 14 countries across the globe report similar illness experiences; although gender, culture, poverty and socio-economic status may act as mediating factors that shape an individual's illness experience. Further research is needed to gain a comprehensive understanding of endometriosis as experienced by women living in low- and middle-income countries.

Keywords: societal burden, endometriosis, infertility, gynaecological service, sexual and reproductive health, women's health, illness experience, meta ethnography, qualitative studies. 


\section{INTRODUCTION}

Endometriosis is defined as the presence of endometrial-like tissue outside the uterus, which induces a chronic, inflammatory reaction (1). Though commonly found in the pelvis, endometriosis has been described in extrapelvic sites including the anterior abdominal wall, surgical scars, diaphragm, omentum, small intestine, appendix, lung, urinary tract, musculoskeletal, and neural systems (2). There is no definite cure for endometriosis, a chronic gynaecological disease associated with pelvic pain and having no clear aetiology or pathophysiology (3). Common treatment options include pain management, hormonal suppression and surgery (1).

Global prevalence rates of the disease are under-estimated (4), possibly because studies on the epidemiology of endometriosis among women living in low-resource countries are lacking. In Africa for example, the true prevalence of endometriosis is under-reported partly due to inadequate facilities and specialized skills in proper assessment of the pelvic area and recognition of the various stages and appearances of the disease by medical professionals (5). Nevertheless, available evidence suggests that endometriosis occurs in women of all race, ethnic and socioeconomic groups; approximately $1 \%$ to $10 \%$ of women of reproductive age - affecting an estimated 176 million women worldwide (4). Endometriosis was found in $30 \%$ of female patients presenting with infertility in Italy (6) and in Nigeria, $48.1 \%$ of women undergoing laparoscopy for gynaecological conditions were diagnosed with endometriosis (7).

Studies have demonstrated that women living with endometriosis experience pain and significant impairments in psycho-social functioning (8), health-related quality of life (HRQoL) and work productivity (4). However, these are mostly quantitative (cross-sectional) studies which utilize general QoL instruments whose psychometric properties and internal consistency are not specific to endometriosis (9). Hereof, this review is aimed at using qualitative research synthesis to achieve a better understanding of patients' perspectives about their illness experiences and make sense of the processes involved.

The strength of a metaethnography is in the systematic identification of key concepts, the comparing of concepts of each study and the translating of studies into one another in a way that represents a conceptual development, which constitutes a fresh contribution to the literature (10). Undoubtedly, the results of this study will contribute some comprehension of the societal burden of endometriosis on women globally. This understanding is useful to address the existing gaps in health service delivery and research; and will help to better cater for the needs of patients with this condition. 


\section{METHODS}

\section{Search Strategy}

The specific research question for this study is: "What are the perceptions of endometriosis patients about the impact of the disease on their lives?" The SPIDER framework was applied in order to create an exhaustive search strategy with clear research concepts (11).

Six electronic bibliographic databases (Medline, CINAHL, Scopus, Embase, Web of Science (WoS) and PsycINFO) and Google Scholar were searched for peer-reviewed papers published in the English Language, from inception of the databases until August 6th 2021.

A comprehensive search strategy was used; applying a combination of keywords, subject headings and free-text terms. To aid the search process, methodological search filters were applied. For example, in CINAHL, additional thesaurus terms for qualitative studies include: interview*, reflexivity or field stud* (12) .

Boolean operators were used to combine search concepts: a variety of search terms within each concept were combined with "or", then results of the combinations within concepts were combined with "and". Search terms were truncated by using wildcards where appropriate, to ensure all relevant articles were returned. The entire search strategies on the respective databases including all keywords, subject headings and combinations are described in Appendices 1,2,3,4,5 and 6.

The search efforts were complemented with reference checking and citation searching. These are considered specifically important to compensate for any inadequacies in retrieval terms (11).

\section{Inclusion and Exclusion Criteria}

To be included in this review, the focus of the study should be on experiences and/or perceptions of endometriosis patients on the impact of endometriosis on their lives, using qualitative methodologies. Primary qualitative research studies are taken to be those which used techniques such as in-depth interviews, semi-structured interviews, focus group discussions (FGDs), observation and reflective diaries to explore participants' experiences (13). Accordingly, all quantitative research studies were excluded: e.g. studies which used techniques such as surveys or questionnaires; cross-sectional or analytical methods. Studies which utilized both quantitative and qualitative methods were excluded if it was not possible to disentangle qualitative pieces from the quantitative.

Studies which had participants who had not been surgically diagnosed with endometriosis were excluded. The study also must have been published in English Language, in a peer-reviewed journal. All the inclusion and exclusion criteria are detailed in Appendix 7. 


\section{Critical Appraisal or Quality Assessment}

The Critical Appraisal Skills Programme (CASP) 2018 qualitative checklist was used to assess the quality of selected papers (14) (see Appendix 9). Comparing quality and methodological techniques of studies proved helpful in assessing the reliability and usefulness of the findings made by the authors.

\section{Data extraction}

A data extraction sheet was prepared on Microsoft Excel. Studies which met quality assessment criteria were examined thoroughly and relevant information from each study was collated on the sheet.

\section{Data synthesis}

Studies were read thoroughly. Noblit and Hare's seven-step process for conducting a meta-ethnography was employed (15); as outlined in Appendix 8. Data was synthesized by Britten's meta-ethnography, an interpretive approach widely used in healthcare research synthesis (10) and themes which consistently appeared in the studies were categorized into key concepts. In this synthesis, the concepts of each study were compared one by one with the key concepts in order to test the extent to which they endorsed or contradicted them. The conclusions of each study were extracted in the form of an explanation, interpretation or description as appropriate. These explanations, interpretations and descriptions were then compared across the whole set of studies. 


\section{RESULTS}

\section{Eligibility}

The systematic search on electronic databases returned 813 papers, the search on Google Scholar returned 62 studies; details of the search results are included in the PRISMA (2009) flowchart (see Figure 1).

After removing 623 duplicates, the inclusion/exclusion criteria were applied, leaving a total of 17 studies. After critical appraisal of the 17 papers using the CASP (2018) checklist, two studies were excluded because their respective aims were not stated at all. Appendix 9 details the quality assessment of the seventeen papers.

\section{Summary}

Fifteen studies were included in the data synthesis, as shown in Table 1. Review included a total of 354 women from fourteen countries (including South Africa, Iran, England, Australia, USA, Sweden, Brazil, China, India, New Zealand), of age range 16-78 years, representing diverse ethnicities (Black, British, Asian, Caucasian, Afro-Caribbean), languages (Afrikaans, English, Portuguese), marital status, socio-economic status and cultures. Participants were recruited from dedicated endometriosis clinics, pain treatment centres, endometriosis support groups and charities (see Appendix 10). All cases of endometriosis must have been surgically diagnosed with a histological confirmation of biopsy. Study participants presented different stages of endometriosis, as classified by the American Society for Reproductive Medicine (1997).

\section{Data Extraction and Synthesis}

Qualitative data were synthesized to develop nine concepts and fifteen second-order interpretations, detailed in Appendix 11. Studies were translated into one another to develop a line of argument and six third-order interpretations, detailed in Table 2. Concepts were found to be overlapping with one another and themes were intricately connected. All the categories were incorporated into a conceptual model, illustrated in Figure $2 b$.

A comprehensive overview of all the key concepts including excerpts of participants' quotes are shown in Appendix 12.

\section{Concepts}

Concepts have been listed in no particular order.

\section{a) Disease symptoms}

The most common symptoms of endometriosis include dysmenorrhea, chronic pelvic pain, dyspareunia, infertility and fatigue. However, the type, pattern, severity and duration of pain varied among women. One woman reported: 
Figure 1 PRISMA (2009) Flow Diagram presenting the number of articles retrieved, included and excluded in this literature review.

Records identified through:
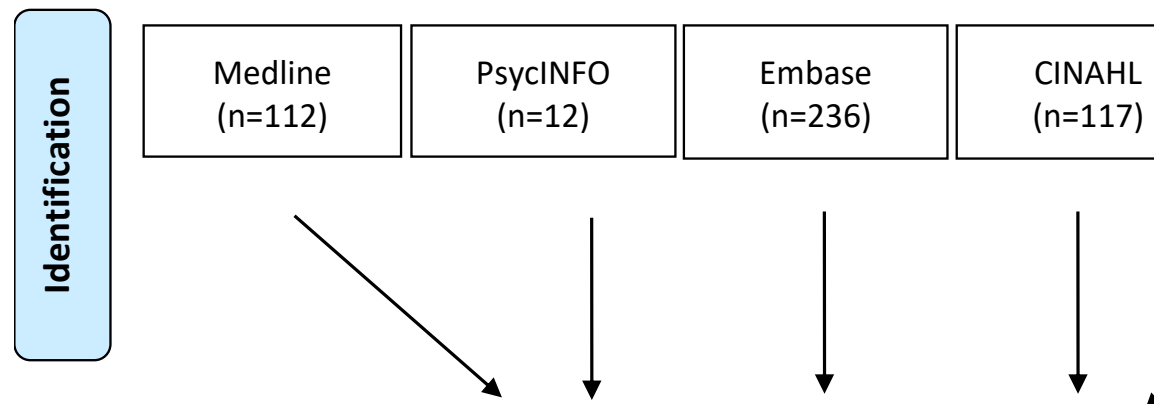

\begin{tabular}{|c|c|}
\hline $\begin{array}{c}\text { WoS } \\
(n=112)\end{array}$ & $\begin{array}{l}\text { ScOPUS } \\
(n=224)\end{array}$ \\
\hline
\end{tabular}

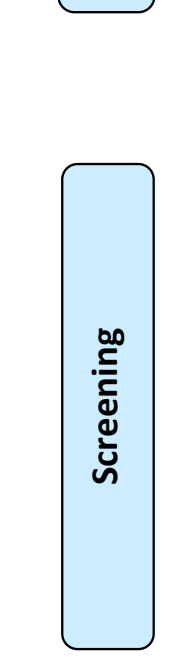

Records after 623 duplicates removed

$$
(n=252)
$$

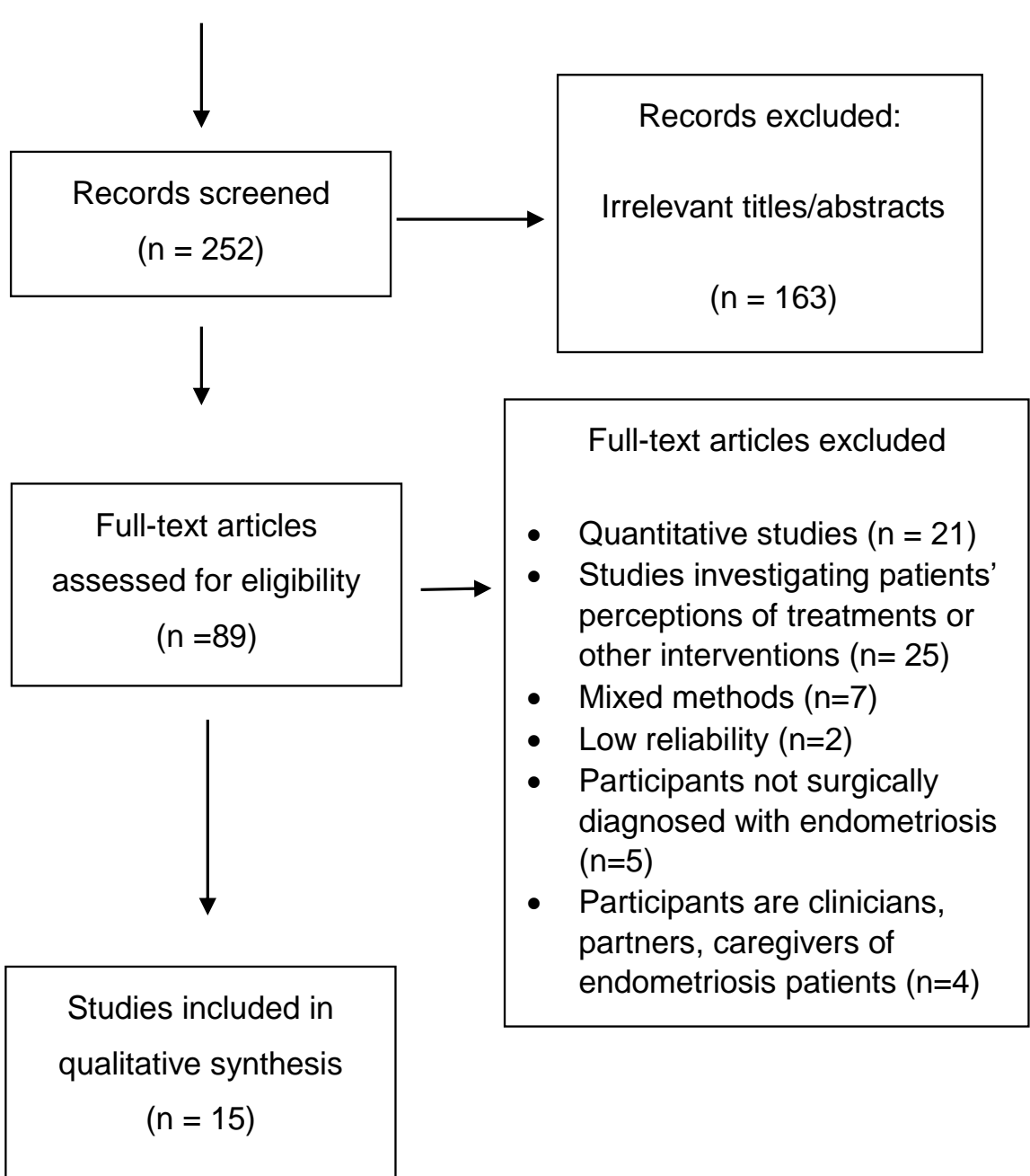


Table 1 Description of included studies

\begin{tabular}{|c|c|c|c|c|}
\hline Author, Year of Publication & Setting & $\begin{array}{l}\text { Participants }(n) \\
\text { Age range in years }\end{array}$ & Data collection & Data analysis \\
\hline Namazi et al., 2021 & Tehran, Iran & $20(23-43)$ & Semi-structured interviews & Content analysis \\
\hline DiBenedetti et al., 2020 & Southern USA & $22(27-48)$ & Indepth interviews & Thematic analysis \\
\hline Hållstam et al., 2018 & Stockholm, Sweden & $13(24-48)$ & Semi-structured interviews & Grounded theory \\
\hline Roomaney \& Kagee, 2018 & Cape Town, South Africa & $25(25-42)$ & Semi-structured interviews & Thematic analysis \\
\hline Mellado et al., 2016 & Southwest Brazil & $29(21-49)$ & Focus group discussions & Grounded theory \\
\hline Hudson et al., 2015 & UK & $22(25-50)$ & $\begin{array}{l}\text { In-depth interviews/semi- } \\
\text { structured interviews }\end{array}$ & $\begin{array}{l}\text { Interpretivist relational approach, } \\
\text { thematic analysis }\end{array}$ \\
\hline Moradi et al., 2014 & Canberra, Australia & $35(17-53)$ & FGDs & Thematic analysis \\
\hline Denny et al., 2009 & Birmingham, England & $27(-)$ & Storytelling/Interviews & Narrative \\
\hline Markovic et al., 2008 & Victoria, Australia & $30(20-78)$ & In-depth interviews & Grounded theory \\
\hline Denny and Mann, 2007 & West Midlands, England & $30(19-44)$ & Semi-structured interviews & Thematic analysis \\
\hline Butt and Chesla, 2007 & California, USA & $13(23-48)$ & Narrative interview & Thematic analysis \\
\hline Huntington \& Gilmour, 2005 & New Zealand & $18(16-45)$ & Interviews & Thematic analysis \\
\hline Jones et al., 2004 & Oxford, England & $24(21.5-44)$ & In-depth interviews & Grounded theory \\
\hline Denny, 2004 & England & $15(-)$ & Interviews & Thematic analysis \\
\hline Cox et al., 2003 & Australia & $61(20-64)$ & FGDs & Thematic analysis \\
\hline
\end{tabular}


"I'm in pain every day. Sometimes it will be in the morning, sometimes in the afternoon, sometimes at night. Sometimes the whole day, especially after I had my period" (16).

b) Health Services Experience

Diagnostic delays, repeated surgeries and recurring disease after treatment are some common experiences amongst study participants. One woman wrote in her diary:

"I had a hysterectomy on 6th December, and now I am regretting this as six months on I am feeling no better at all, in fact I feel worse" (17).

Another reported: "I have undergone surgery several times, but my illness is recurring" (18).

To address this more holistically, two sub-concepts were created as follows: 1) delayed diagnosis 2) patients' perception about gynaecological services. Within these sub-concepts are important points illustrated in Figure 2.

c) Isolation and limited social participation:

Participants reported cancelling or modifying social plans due to endometriosis-associated fatigue and pain. A woman described herself as "Lethargic, more just tired, I mean just feeling drained. No mental clarity." (19).

Chronic pain, heavy bleeding, weight gain, dyspareunia and struggles in achieving pregnancy contributed negatively to patients' self-image, thus leading to isolation. One woman said:

"Overall endometriosis has made me live a very solitary life" (20).

Another participant commented, "My relatives always avoid my company. No one understands me" (21).

Many of the women described feeling worried about the pain episodes starting in public because they did not want to feel embarrassed, hence stayed at home.

d) Limited physical functioning

The difficulties with mobility experienced by patients (i.e. walking, cooking, dressing up, bathing, standing, bending, driving, sitting, lifting or exercising) are associated with pain and fatigue, inevitably resulting in their inability to fulfil roles.

"But I feel ... limited is the word, I think. You feel limited and not free, somehow... You sort of want to do things and be active and that part of me is no longer there..." (21)

e) A Coterie of Emotions

Diverse emotions characterized the endometriosis illness experience among women in all the selected studies. Feelings of fear, uncertainty, helplessness, anxiety, anger, despair, worry, sadness, selfconsciousness, worthlessness, low self-esteem, shame, guilt, grief, regret, confusion, isolation, 
frustration, hopelessness, misery, distress, despair, moodiness, short temperament and embarrassment were noted in relation to their endometriosis. These emotions are known to have an unfavourable psychological impact on the women and their productivity. Some women reported depression and suicidal ideation $(16,22)$. A 23-year old South-African woman recounted her sadness in the statement:

"...for a long time, I felt ... this deep, deep, deep sadness ... it's as if there's this heaviness over me... It feels like I can't overcome it. (It is) like a dark cloud or something" (16).

An Iranian woman described her emotions as follows: "I did not like to be alive, I was very annoyed, [...] It was so hard to breathe, I wanted to die". (18)

Women expressed fear and worry over infertility, disease recurrence, disease prognosis, interference with career, education and employment, finances and high cost of treatment (e.g. in-vitro fertilization, excision surgery), sexual/marital life, motherhood responsibilities, finding a new partner (due to dyspareunia), passing endometriosis on to a future daughter and disease progressing to cancer.

Though many patients had negative emotions, a few demonstrated optimism and hope. Positive emotions were connected to satisfaction with quality of care being received and good coping mechanisms. One woman said:

"I do finally feel more hopeful, and I think it's just that the energy levels are coming back, and I don't think I've had energy levels like I've got now for years" (17).

f) Coping strategies \& Support

Supportive partners, families, friends, employers and patient groups were helpful to the women who had them.

"He [partner] helps me a lot. Usually in the morning I need him. He has to help me out of bed because I'm in pain" (23).

Participants reported using a combination of coping strategies to manage endometriosis and the effects of its symptoms on their HRQoL. These include alternative therapies (acupuncture, Chinese herbs); dietary changes (with emphasis on a caffeine-free diet with a high vegetable and fruit content); accepting the disease and advocating for themselves, joining local support groups, acquiring knowledge about endometriosis, scheduling social and work activities around menstrual cycle, limiting physical activity, adopting an optimistic attitude and evoking spirituality $(16,17,20,21,22,24,25)$.

A woman speaking about the effect of aloe vera juice, said:

"I've got energy now, I'm positive and it's so lovely not having pain" (25).

On the other hand, some women reported negative effects of endometriosis on their lifestyle such as becoming opioid-dependent, smoking more tobacco cigarettes, consuming more alcohol, and in two cases using illicit drugs to help them cope with pain, insomnia or sadness (20). 
Table 2 Synthesis; including concepts, second-order and third-order interpretations

\begin{tabular}{|c|c|c|}
\hline Concepts & Second-order interpretations & Third-order interpretations \\
\hline $\begin{array}{l}\text { 1) Disease Symptoms: dysmenorrhea, CPP, } \\
\text { painful sex, fatigue, pains, nausea. } \\
\text { 2) Health Services Experience: delayed } \\
\text { diagnosis, patients' perception about } \\
\text { gynaecological services. } \\
\text { 3) Isolation and limited social participation } \\
\text { 4) Limited physical functioning } \\
\text { 5) Coterie of emotions } \\
\text { 6) Sex and intimate relationships } \\
\text { 7) Infertility } \\
\text { 8) Coping strategies/Support } \\
\text { 9) Work life and Education }\end{array}$ & $\begin{array}{l}\text { a) Living with severe painful endometriosis } \\
\text { signified a struggle for coherence } \\
\text { b) Understanding patient experience is } \\
\text { valuable in assessing the needs of patients, } \\
\text { and improving patients' well-being. } \\
\text { c) The patient's illness experience is shaped by } \\
\text { mediating factors like gender, ethnicity and } \\
\text { being in a relationship. } \\
\text { d) Women's socio-demographic background, } \\
\text { family health history and wider social beliefs, } \\
\text { shaped illness experiences. } \\
\text { e) Miscommunication between women and } \\
\text { HCPs, absence of adequate information } \\
\text { concerning disease, and subsequent } \\
\text { frustration lead to social isolation. } \\
\text { f) Better understanding of the long term and } \\
\text { wide-ranging impact of endometriosis on } \\
\text { women's lives could be useful in improving } \\
\text { patient's life experiences. } \\
\text { g) Gendered notion about endometriosis is } \\
\text { integral to the uncertainty around the } \\
\text { disease. }\end{array}$ & $\begin{array}{l}\text { i. Women living with endometriosis } \\
\text { experience a wide range of symptoms } \\
\text { including CPP, dysmenorrhea, } \\
\text { dyspareunia, infertility and fatigue. } \\
\text { However, the disease presents differently } \\
\text { in patients; as location, intensity and } \\
\text { duration of pains vary. } \\
\text { Women experience diagnostic delays for } \\
\text { a number of reasons, including the } \\
\text { gendered notion of the disease and } \\
\text { inadequate knowledge } \\
\text { endometriosis among primary HCPs. } \\
\text { Current treatment options for } \\
\text { endometriosis have drawbacks and are } \\
\text { not equally effective for all patients. } \\
\text { Diagnostic delays, persistent symptoms, } \\
\text { scanty information to patients about the } \\
\text { disease, side effects of hormonal and } \\
\text { surgical treatment, treatment costs, all } \\
\text { intersect into a web of struggles and } \\
\text { incoherence for patients. }\end{array}$ \\
\hline
\end{tabular}




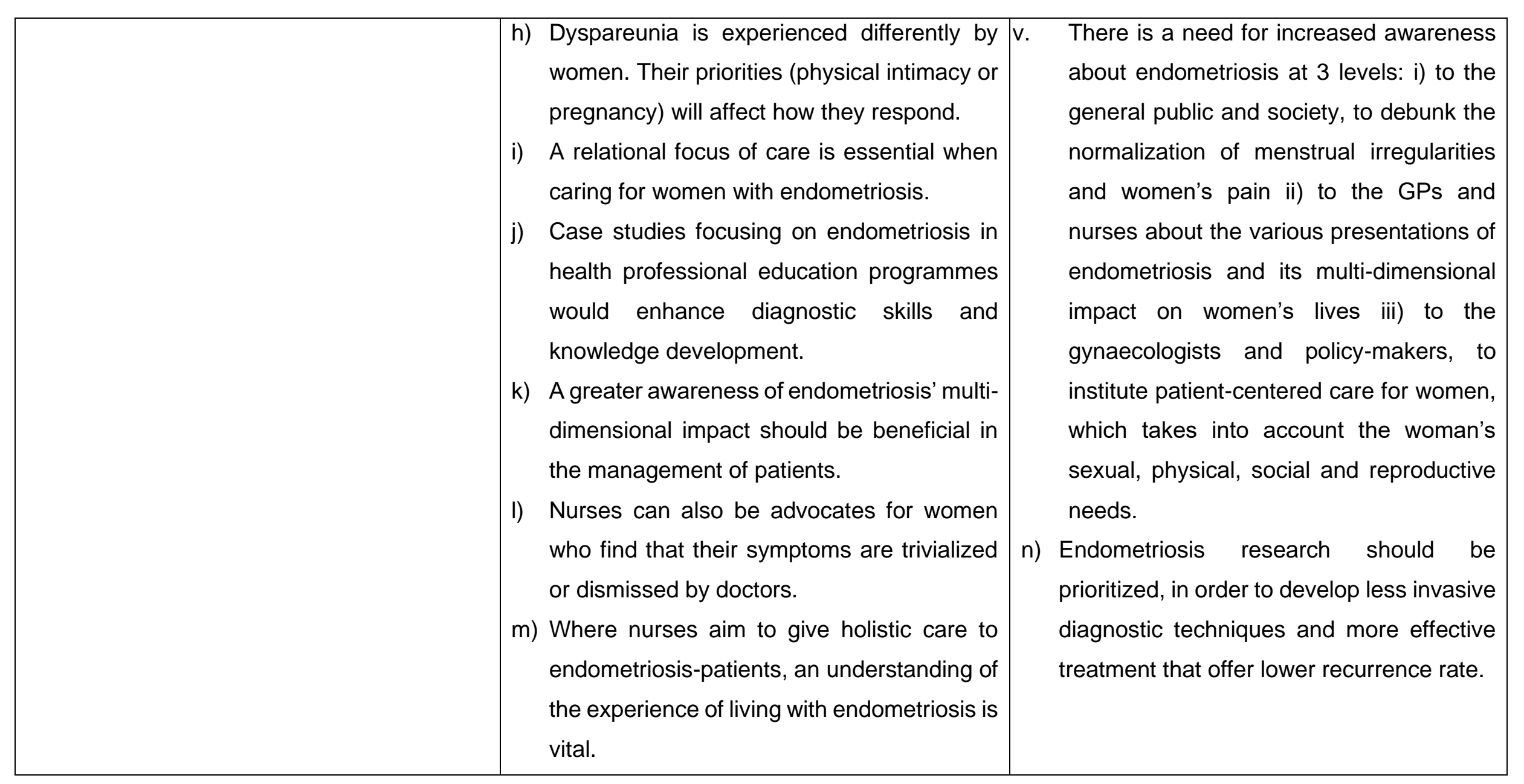


g) Sex and Intimate Relationships

Painful intercourse, fear associated with intercourse and avoidance of intercourse contributed to conflict in romantic relationships and eventual break-up in some.

"You're with the person you love and ... You're having sex and you have to stop because it starts hurting" (26).

The importance placed on the absence of sex varied for the study participants. While some women commented on the supportive stance of their partners, others felt their partners were suspicious of their reluctance to be sexually active $(23,26,27)$.

h) Infertility

Across cultures, the stigma associated with infertility was evident in the concerns expressed by the participants. Women who struggled to conceive stated that their infertility was detrimental to their marital harmony. A 28-year old woman expressed her insecurity in the statement:

"[...] what if he leaves me for another woman because I can't give him a child"? (16)

Another participant remarked on how endometriosis affected her marriage: "have been married and the marriage broke up...because I couldn't fall pregnant plus I guess I was moody and in a lot of pain and he couldn't put up with that..." (20).

i) Work life and Education

Women who did not lose their jobs lived in fear of losing their jobs because of absenteeism. Others gave up sports, deferred university, performed badly in their academics, dropped out of school, were passed up for promotion or remained in non-challenging job roles because of the disease - more details in Appendix 13.

"Couldn't work for a year after the surgery. Had to sell business, didn't have the energy to advertise so didn't get much for it" (28).

"On those bad days when I'm at work, it's hard to focus during those days, and so I would say sometimes I do leave the office early because I'm useless" (19). 


\section{Figure 2}

a) Health Services Experience Concept

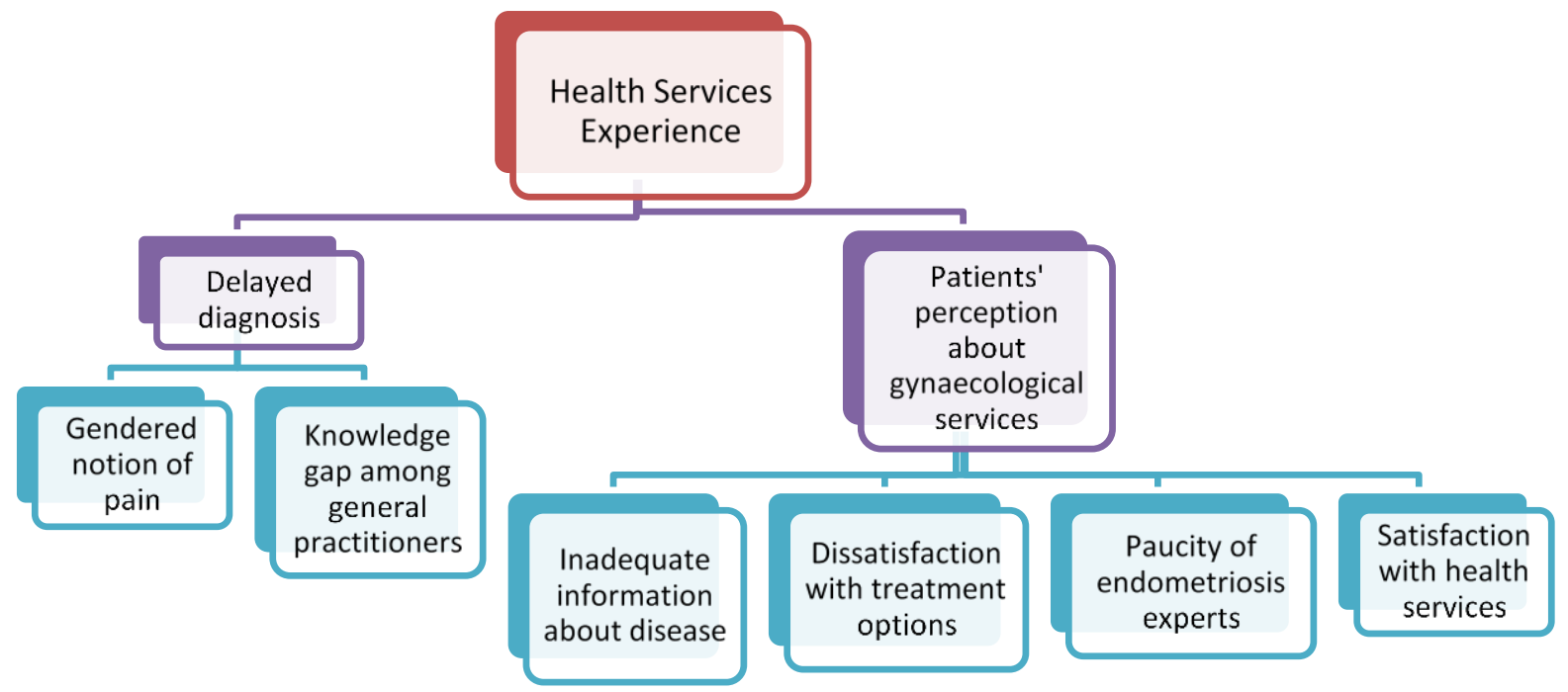

b) Conceptual model for the impact of endometriosis on patients' lives

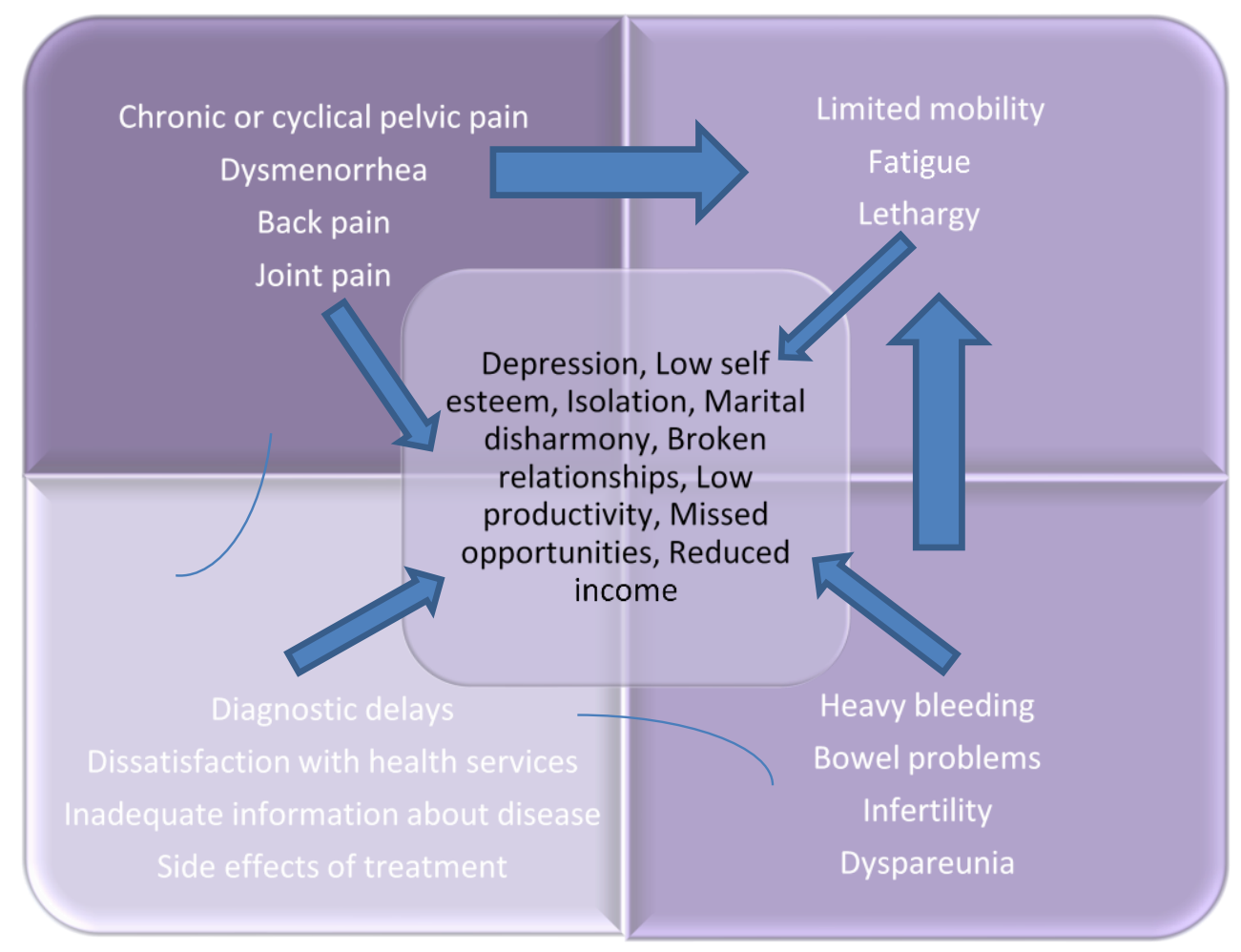




\section{DISCUSSION}

Our study results have established that women with endometriosis encounter a number of problems categorized into nine broad concepts encompassing but not limited to: infertility, difficulty in maintaining intimate relationships with their partners, psychological distress, negative emotions which may lead to suicidal ideations and a low sense of self. Additionally, low productivity at work, increased absenteeism, poor academic performance, social isolation and a low quality of life are associated with endometriosis.

The findings of this study include a conceptual framework connecting the different experiences of women with endometriosis, as illustrated in Figure $2 \mathrm{~b}$. This model was designed to depict how all the aspects of the illness experience interact to create a web of struggles and incoherence for the women; including diagnostic delay, disease symptom(s) and its effect on her fertility, relationships, productivity, and social participation. For example, a woman with endometriosis experiencing infertility, chronic pain, fatigue and heavy bleeding may have these symptoms interfere with her ability to fulfil roles and meet up with her financial, social or other obligations. Her inability to live a normal life as revealed by our study, may lead to a low self-esteem, depression, isolation, unproductivity or suicidal ideations.

To the best of our knowledge, this is the first meta-ethnography investigating the impact of endometriosis on patients' quality of life. It is also the first review of qualitative literature on the illness experiences of endometriosis patients, which includes a study from Africa. We acknowledge that qualitative research findings are mostly contextually specific. However, our findings show that women with endometriosis have somewhat similar illness experiences though culture, ethnicity, race, gender, and relationship status may act as mediating factors to shape women's illness experience.

One such similarity is the diagnostic delay experienced by endometriosis patients. A survey of 228 women in the United Kingdom and USA uncovered the mean length of time between onset of symptoms and diagnosis to be 7.96 years (29). A narrative review (30) noted that the average duration of diagnostic delay was 6.27 years. The multicentre cross-sectional study involving 1,418 women from ten countries noted that delay was longer in women with more 'pelvic' symptoms (e.g. CPP, dysmenorrhea and dyspareunia), a higher body mass index and at clinics delivering predominantly state-funded healthcare (4) .

Reasons for diagnostic delays in endometriosis patients have been discussed extensively in the literature. Seear (2009) suggested that women consciously conceal their menstrual anomalies through practices of the 'menstrual etiquette' (31). Furthermore, endometriosis mimics other conditions like irritable bowel syndrome, pelvic inflammatory disease and appendicitis (32). Given the diverse localizations and forms of the disease, its heterogeneous presentation of symptoms commonly leads to misdiagnosis and dismissal. Consequently, the woman's verbal narration of pain and her assertiveness in the face of dismissals are pivotal to eventual diagnosis as the condition rarely manifests in external, visible symptoms.

Laparoscopy is the gold standard for the diagnosis of endometriosis (2). Owing to the heterogeneous and sometimes subtle appearance of implants, the accuracy of diagnosis depends on the ability of the surgeon to identify the disease (5). In low- and middle-income settings like sub-Saharan Africa, access to laparoscopy for diagnosis of endometriosis is limited (7) and inadequate facilities and specialized skills for 
adequate assessment of the pelvis and recognition of the various types and appearances of the disease may lengthen the diagnostic delay among women.

Living with chronic illness can interrupt a person's life and may bring disarray into a person's hopes and plans; creating an abnormal, sometimes damaging shift from an otherwise predictable life - as is obtainable with endometriosis (33). In the case of endometriosis-associated infertility for example, cultural dialogues insist that parenthood is a normal and expected aspect of adult life - childlessness therefore, may constitute a significantly disruptive course. One study (34) reported that endometriosisassociated infertility is of particular concern to women from minority ethnic groups and revealed that social expectations of motherhood and fertility can influence how women experience endometriosis, owing to its implications on reproduction. Women therefore, bear endometriosis-associated infertility and the inability to fulfil the normative expectations of sexual intimacy in their relationships; with feelings of failure, responsibility, and guilt.

A previous systematic review (35), reported that very few studies investigated women's accounts of actual or anticipated infertility: an observation which differs from the findings of this review. It is therefore noteworthy that seven out of the fifteen selected papers reported elaborately on patients' experiences with infertility and gave clear insight into its effects on women's identity, intimate relationships and expectations for the future.

Another important theme identified in this meta-ethnography is the individual/household financial burden of managing endometriosis. However, this point was elucidated in just two studies $(16,20)$, hence it is unclear whether this issue was unimportant to the research participants in the other selected studies or whether the research approach did not elicit such accounts.

The financial constraint in managing endometriosis needs to be given more attention especially in low and middle-income countries (LMICS) where out-of-pocket payments (OOP) remains the mainstay of healthcare funding, constituting above $90 \%$ of private expenditures on health (33). In Nigeria for example, the introduction of user fees in public health facilities led to a progressive increase in healthcare cost to consumers and limits some households from seeking timely healthcare services (34). Hesitancy towards care-seeking due to fear of incurring costs could further delay the diagnosis and treatment of endometriosis because patients of low socio-economic status and/or a lower level of education may face barriers in accessing specialized care; a similar observation was made in a population-based study in Israel (35). Arguably, this study therefore, has recommended a need for further research to estimate the socioeconomic cost of the disease to enable health policy-makers to provide better care and support these patients.

With respect to all mentioned above, this study has policy implications and calls for more efforts to address the social, emotional, and economic burden that is borne by persons with endometriosis. We strongly recommend that endometriosis care delivery should be sensitive, holistic, relational and patientcentered to manage the multi-dimensional, pervasive, and disruptive impact of the disease. Stakeholders such as policy makers, community leaders, employers and school administrators should be sensitized about endometriosis and its impact on sufferers. It is also necessary that healthcare facilities and 
healthcare workers are provided with the right resources which will facilitate the early diagnosis of endometriosis and reduce the OOP associated with managing the condition. This will make it much easier for young girls and women to manage the condition and improve their quality of life while living with endometriosis.

In view of the qualitative nature of our study, one of the study limitations is that the findings reported may have been affected by the reflexivity of the authors of the included studies and that of the authors who conducted this review. Another limitation is that the strict inclusion criteria used may have caused the perspectives of women with endometriosis in excluded articles to be missed, thus affecting the results obtained. The strength of this study lies in its broad scope that ensured representation of diverse cultures, ethnicities, and countries. Also unique to this research is the application of Britten's meta-ethnography to develop a conceptual model that paints a picture about the experiences of endometriosis patients from across the globe. 


\section{CONCLUSION}

This review has shown similarities in the illness experiences of endometriosis patients, cutting across disease stage, nationality, geographical location, ethnicity, culture and language. Further research should be aimed at a comprehensive understanding of endometriosis as experienced by women living in low-and middle-income countries; and also, to estimate the societal costs of the disease. This will enable health systems to provide better care and support for patients and their care-givers.

\section{Acknowledgement}

The authors appreciate Dr. Aniebet-Abasi Inemesit Akpan (NAIJAHEALTH INITIATIVE, and Kharkiv National Medical University, Ukraine), Dr. Ebi Ekpokiri (University of Chester and State Ministry of Health, Imo state, Nigeria), Dr Ekow Adom Mensah (National Health Service, London), Dr Lamin B. Sonko (United Nations - UN), Wendy Bingham (Extrapelvic Not Rare) and Prof. Nataliia Gavkalova (Kharkiv National University, Ukraine) for their insightful opinion on the final draft of this article.

\section{Authors Contribution}

OCN conceptualized the study, designed the methodology of the study, solely conducted the search for literature, solely wrote the first draft and contributed to the final draft of the manuscript. IOI contributed to the methodological design of the study, conducted a scoping review and revised the first draft of the manuscript and contributed to the final draft of the manuscript. FIA contributed to the final draft of the manuscript. UO contributed to the final draft of the study. All authors reviewed and accepted this final draft of the manuscript.

\section{Competing Interests}

The authors have no competing interests to declare. OCN is the Founder of EndoSurvivors International Foundation; a non-profit in Nigeria focused on endometriosis awareness, education, research and advocacy.

\section{Funding}

No funding was received for this study. 


\section{BIBLIOGRAPHY}

1. Kennedy S, Bergqvist A, Chapron C, D'Hooghe T, Dunselman G, Greb R, et al. ESHRE guideline for the diagnosis and treatment of endometriosis. Hum Reprod. 2005 Oct;20(10):2698-704.

2. Kim, A, Adamson, G, Glob. libr. women's med., (ISSN: 1756-2228) 2008; DOI 10.3843/GLOWM.10011

3. Ballard K, Lowton K, Wright J. What's the delay? A qualitative study of women's experiences of reaching a diagnosis of endometriosis. Fertil Steril. 2006 Nov;86(5):1296-301.

4. Nnoaham KE, Hummelshoj L, Webster P, d'Hooghe T, de Cicco Nardone F, de Cicco Nardone C, et al. Impact of endometriosis on quality of life and work productivity: a multicenter study across ten countries. Fertil Steril. 2011 Aug 1;96(2):366-373.e8.

5. Kyama CM, Mwenda JM, Machoki J, Mihalyi A, Simsa P, Chai DC, et al. Endometriosis in African women. Womens Health (Lond Engl). 2007 Sep;3(5):629-35.

6. Prevalence and anatomical distribution of endometriosis in women with selected gynaecological conditions: results from a multicentric Italian study. Gruppo italiano per lo studio dell'endometriosi. Hum Reprod. 1994 Jun;9(6):1158-62.

7. Fawole AO, Bello FA, Ogunbode O, Odukogbe A-TA, Nkwocha GC, Nnoaham KE, et al. Endometriosis and associated symptoms among Nigerian women. Int J Gynaecol Obstet. 2015 Aug;130(2):190-4.

8. Gao X, Yeh Y-C, Outley J, Simon J, Botteman M, Spalding J. Health-related quality of life burden of women with endometriosis: a literature review. Curr Med Res Opin. 2006 Sep;22(9):1787-97.

9. Jia S-Z, Leng J-H, Shi J-H, Sun P-R, Lang J-H. Health-related quality of life in women with endometriosis: a systematic review. J Ovarian Res. 2012 Oct 18;5(1):29.

10. Britten N, Campbell R, Pope C, Donovan J, Morgan M, Pill R. Using meta ethnography to synthesise qualitative research: a worked example. J Health Serv Res Policy. 2002 Oct;7(4):209-15.

11. Cooke A, Smith D, Booth A. Beyond PICO: the SPIDER tool for qualitative evidence synthesis. Qual Health Res. 2012 Oct;22(10):1435-43.

12. Wilczynski NL, Marks S, Haynes RB. Search strategies for identifying qualitative studies in CINAHL. Qual Health Res. 2007 May;17(5):705-10.

13. Jones G, Jenkinson C, Kennedy S. The impact of endometriosis upon quality of life: a qualitative analysis. J Psychosom Obstet Gynaecol. 2004 Jun;25(2):123-33.

14. https://casp-uk.net/wp-content/uploads/2018/01/CASP-Qualitative-Checklist-2018.pdf [Internet]. [cited 2021 Sep 8]. Accessed August 8th 2021

15. Noblit G, Hare R (1988). Meta-ethnography: synthesizing qualitative studies. . 11th ed. G N, editor. London: Sage Publications.; 1988.

16. Roomaney R, Kagee A. Salient aspects of quality of life among women diagnosed with endometriosis: A qualitative study. J Health Psychol. 2018 Jun;23(7):905-16. 
17. Denny E. Women's experience of endometriosis. J Adv Nurs. 2004 Jun;46(6):641-8.

18. Namazi M, Behboodi Moghadam Z, Zareiyan A, Jafarabadi M. Exploring the impact of endometriosis on women's lives: A qualitative study in Iran. Nurs Open. 2021 May;8(3):127582.

19. DiBenedetti, D., Soliman, A.M., Gupta, C. et al. Patients' perspectives of endometriosis-related fatigue: qualitative interviews. J Patient Rep Outcomes 4, 33 (2020).

20. Moradi M, Parker M, Sneddon A, Lopez V, Ellwood D. Impact of endometriosis on women's lives: a qualitative study. BMC Womens Health. 2014 Oct 4;14(1):123.

21. Mellado, B. H., Falcone, A. C. M., Poli-Neto, O. B., Rosa e Silva, J. C., Nogueira, A. A., \& Candido-dos-Reis, F. J. (2016). Social isolation in women with endometriosis and chronic pelvic pain. International Journal of Gynecology \& Obstetrics, 133(2), 199-201

22. Cox H, Henderson L, Andersen N, Cagliarini G, Ski C. International Journal of Nursing Practice 2003;9:2-9

23. Strzempko Butt F, Chesla C. Relational patterns of couples living with chronic pelvic pain from endometriosis. Qual Health Res. 2007 May;17(5):571-85.

24. Hållstam A, Stålnacke BM, Svensén C, Löfgren M. Living with painful endometriosis - A struggle for coherence. A qualitative study. Sex Reprod Healthc. 2018 Oct;17:97-102.

25. Markovic M, Manderson L, Warren N. Endurance and contest: women's narratives of endometriosis. Health (London). 2008 Jul;12(3):349-67.

26. Denny, E., \& Mann, C. H. (2007). Endometriosis-associated dyspareunia: the impact on women's lives. Journal of Family Planning and Reproductive Health Care, 33(3), 189-193.

27. Hudson N, Culley L, Law C, Mitchell H, Denny E, Raine-Fenning N. "We needed to change the mission statement of the marriage": biographical disruptions, appraisals and revisions among couples living with endometriosis. Sociol Health Illn. 2016 Jun;38(5):721-35.

28. Huntington A, Gilmour JA. A life shaped by pain: women and endometriosis. J Clin Nurs. 2005 Oct;14(9):1124-32.

29. Hadfield R, Mardon H, Barlow D, Kennedy S. Delay in the diagnosis of endometriosis: a survey of women from the USA and the UK. Hum Reprod. 1996 Apr;11(4):878-80.

30. Culley L, Law C, Hudson N, Denny E, Mitchell H, Baumgarten M, et al. The social and psychological impact of endometriosis on women's lives: a critical narrative review. Hum Reprod Update. 2013 Dec;19(6):625-39.

31. Seear K. The etiquette of endometriosis: stigmatisation, menstrual concealment and the diagnostic delay. Soc Sci Med. 2009 Oct;69(8):1220-7.

32. Ballard K, Lane H, Hudelist G, Banerjee S, Wright J. Can specific pain symptoms help in the diagnosis of endometriosis? A cohort study of women with chronic pelvic pain. Fertil Steril. 2010 Jun;94(1):20-7.

33. Bury, M. (1982). Chronic illness as biographical disruption. Sociology of Health and IIIness, $4(2), 167-182$

34. Denny, E., Culley, L., Papadopoulos, I., Apenteng, P., et al. (2011). From womanhood to 
endometriosis: findings from focus groups with women from different ethnic groups, Diversity in Healthcare, 8, 3, 167-80.

35. Young K, Fisher J, Kirkman M. Women's experiences of endometriosis: a systematic review and synthesis of qualitative research. J Fam Plann Reprod Health Care. 2015 Jul;41(3):225-34.

36. Chernichovsky D, Meesook OA. Utilization of health services in Indonesia. Soc Sci Med. 1986;23(6):611-20.

37. Onwujekwe O, Uzochukwu B. Socio-economic and geographic differentials in costs and payment strategies for primary healthcare services in Southeast Nigeria. Health Policy. 2005 Mar;71(3):383-97.

38. Eisenberg VH, Weil C, Chodick G, Shalev V. Epidemiology of endometriosis: a large populationbased database study from a healthcare provider with 2 million members. BJOG. 2018 Jan;125(1):55-62. 